\title{
An Approach to Develop a Generative Design Technology for Power Systems
}

\author{
Serge Protasovitch Kovalyov \\ V.A. Trapeznikov Institute of Control Sciences of Russian Academy of Sciences \\ Moscow, Russia \\ kovalyov@energy2020.ru
}

\begin{abstract}
The generative design technology for power systems is proposed for automatic (rather than automated) efficiency analysis and generation of design decisions in the processes of constructing and renovating critical power supply infrastructure. The key constituents and some supporting software tools to implement the technology are identified.
\end{abstract}

Keywords—generative design; power system; energy management; multi-objective optimization; design space; requirements engineering; co-modeling data and processes

\section{INTRODUCTION}

Generative design is naturally considered as the next stage of industrial products design automation. In essence, generative design aims to pass generation of efficient design and technological decisions to the computer, eliminating human intervention. Contrast this intention with design documentation and verification that are the only engineering activities properly supported in the majority of commercially available CAD and PLM software products as of the first decade of the XXI century. The key steps of a generative design technology include transformation of requirements to a product into a multi-objective optimization problem, particularly topological, and subsequent implementation of the best solution by means of automatic manufacturing, particularly 3Dprinting [1].

Such an approach to design is apparently relevant in application to power systems. Indeed, it is entirely reasonable to consider the power system as a complex industrial product with potentially infinite life cycle. Designing such large-scale critical infrastructure as a power system is a labor-intensive multi-variant process that can be greatly improved by automation. However, the progress in such automation is significantly lagging behind technologically advanced manufacturing sectors: aerospace, electronics and the likes [2]. Some years ago, introduction of software intensive life cycle support technologies (generally known as CALS) into the design and operation of power systems had required a significant amount of time and deep conceptual studies [3]. The adoption of generative design undoubtedly also requires innovative approaches to digital representation and analysis of power systems, especially given that methods that are efficient in mechanical engineering (like topological optimization of shape and 3D- printing) are not applicable to energy facilities. In particular, technological processes of constructing and renovating power systems are of the assembly nature. Hence, novel powerful intellectual discrete optimization algorithms are needed that are capable to find topological and configuration solutions in a complex space of discrete non-numeric assembly-type design variables. The present paper aims to identify relevant approaches to develop these algorithms and other constituents of an innovative software tool for generative design of power systems.

The paper is composed as follows. In Section II, general principles of generative design of power systems are elaborated. Section III discusses requirements engineering for power systems as a key premise to any design automation. Some conclusions are drawn in Section IV.

\section{Generative Design PRINCIPLES FOR POWER SYSTEMS}

The overall generative design process is shown on Fig. 1 [1]. During the first step (inspire), the engineer specifies the requirements, objective functions, and constraints to the computer using a language that is natural in the product application domain. Then, the computer automatically generates a (potentially very large) number of design alternatives and in cooperation with the engineer explores their properties to estimate the degree of compliance with the requirements and proximity to the optimum wrt the objective functions. These steps are repeated until a final satisfactory design solution is available, which is passed to automatic fabrication. To date, this approach has been successfully tested at design of certain parts of dependable structures in machine building industry. Notorious examples include a satellite radio antenna, a race car roll hoop, an airbus cabin partition, etc.

However, design at the level of parts is irrelevant for power systems: here, the computer should generate and explore alternatives for the architecture, topology, types and characteristics of the equipment, grid modes, and control algorithms. The search for (sub-, Pareto)optimal alternatives is performed in the design space that is automatically constructed on the basis of stakeholder requirements. Navigation in such a space is extremely complex, since it contains not only numerical variables ("coordinate axes"), but also discrete non-numerical variables that take values as electrical grid graphs, equipment database records, 


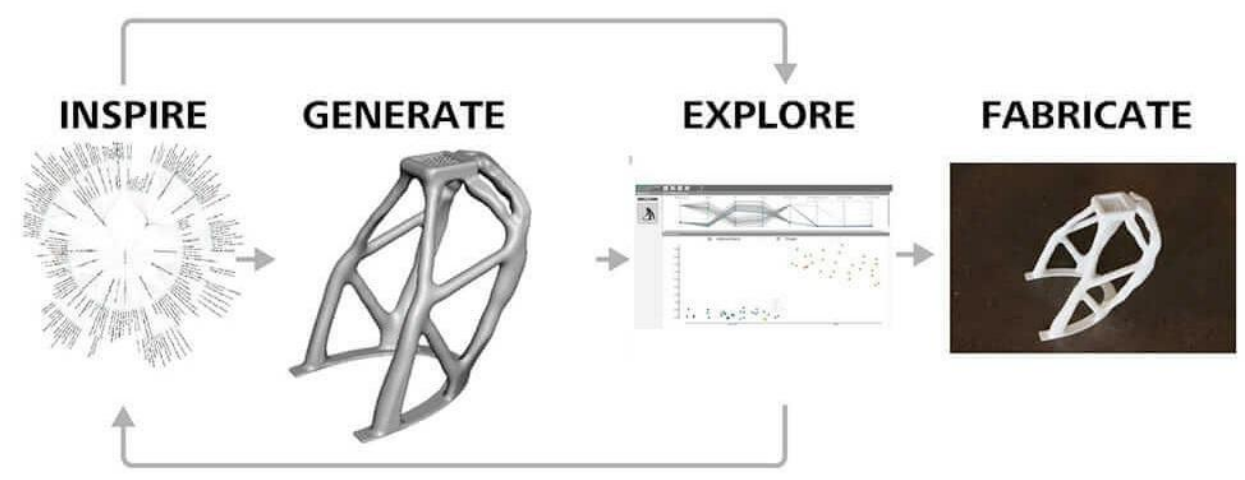

Fig. 1. The generative design process [1].

control algorithm charts, etc. The navigation complexity explosively increases with the growth of the energy facility size. As a promising mathematical tool to describe and explore such design spaces, category theory is proposed [4]. Alternative power system configurations are represented by diagrams in the suitable category with formal models of system constituents as objects and integration relations as morphisms. Morphisms among diagrams are then derived along which the navigation in the diagram space can be performed efficiently.

Nontrivial algorithms also occur at calculating various objective functions of the design optimization problems: minimum discounted costs, maximum infrastructure reliability, etc. Examples available in the literature present solutions to rather simple optimization problems of this kind. For instance, consider the selection of optimal points to connect reactive power compensation devices to the electrical grid [5]. The design variables that comprise the design space are the number, the nominal power, and possible locations of the devices. Minimum discounted costs is employed as an objective function, so operational expenses for each equipment unit need to be estimated. Permissible values of currents and voltages are imposed as constraints, so evaluation and analysis of the steady-state grid mode is required. Although calculation of the objective function is so much nontrivial, this optimization problem is shown to be solvable by "lightweight" algorithms such as a coordinate-wise descent or a branch-and-bound algorithm.

More involved optimization problems occur at designing hybrid renewable energy systems (HRES) where renewable resources (solar, wind, biogas) are backed up by energy storage and conventional fuelconsuming generators. Consider the following example of such a problem [6]. The HRES is constructed by stacking together devices of four kinds: photovoltaic panels, wind turbines, batteries, and diesel generators. All devices of the same kind are assumed identical, which allows representing the HRES configuration as a vector of integer numbers. Given an area with known load demand and weather conditions, an optimal HRES design is sought that minimizes three objective functions: the annualized cost of system, the loss of power supply probability, and the fuel emissions. Numbers of devices of each kind along with installation parameters (such as a solar panel tilt angle) are used as design variables. Constraints determine reasonable bounds for variables. This multi-objective problem is solved by the preference-inspired coevolutionary algorithm using goal vectors (PICEA-g). The algorithm generates a set of non-dominated solutions from which the designer can select the desired one.

In contrast with the toy examples, the full-fledged generative design technology for critical power infrastructure requires novel intellectual discrete optimization methods, including those based on evolutionary genetic algorithms with self-learning, generative adversarial neural networks (GAN, which were recently employed in conventional generative design of machine parts [7]), etc. In order to evaluate the properties of alternative configurations generated by the algorithms, it is reasonable to feed them into the existing power systems analysis and simulation software tools. Examples of such tools include Power Digital from General Electric (USA), PowerFactory from DIgSILENT (Germany), AMIGO from RTSoft (Russia), EnergyCS from CSoft (Russia). Configurations approved by the analysis performed in the software are then passed for validation to hardware-in-the-loop and in situ testing installations. The best configuration is ultimately selected as the target design of the power system. In order to automate its implementation, assignments for mobile industrial robots and settings of control devices are derived from the configuration.

\section{ENGINEERING REQUIREMENTS FOR POWER SYSTEMS}

The success of the design process depends critically not only upon correct and fast solution of optimization problems but also upon correct statement thereof. As mentioned above, in the generative design cycle, design variables, objective functions, and constraints for optimization problems are automatically retrieved from stakeholder requirements. To enable the retrieval, all requirements should be converted to a machine readable format and consolidated in a shared database. The format should be developed taking into account different standard notations for different kinds of requirements. Notable 
example is the XML notation provided in the IEC standard 61970-301:2016 "Energy management system application program interface (EMS-API) Part 301: Common information model (CIM) base" to describe the power equipment and grid topology. Requirements can be represented in different forms, yet means of automatic verification of digital models of candidate power system configurations against them should always be available. For example, load requirements can be specified in the traditional form of a list of power consumption units with their capacity characteristics and typical operation modes. Alternatively, a representative set of load profile samples can be provided to train a suitable predictive neural network.

In order to populate the requirements database of the desired kind, the full cycle of requirements engineering according to the ISO/IEC/IEEE standard 29148:2018 "Systems and software engineering-Life cycle processes-Requirements engineering" should be performed. In the course of power systems construction and renovation, the cycle includes a number of complex multi-step energy management processes: energy policy elaboration, technical documentation analysis, energy audit, implementation of instrumental measurement of actual production and consumption, etc. These processes are governed by the ISO standard 50001:2011 "Energy management systems-Requirements with guidance for use". Hence, essential activities prescribed there and in the ISO/IEC/IEEE 29148:2018 standard should be combined by means of computer assistant tools. Upon performing these combined activities, a number of overlapping views (representations) of the power system by different stakeholders are iteratively constructed and recorded. The target set of requirements is obtained from these views by analyzing (and correcting as needed) in order to gain completeness, consistency, affordability, and boundedness (relevance). Each requirement is supplied with enough metadata allowing to trace its evolution and satisfaction in the course of energy management processes.

Given that generative design algorithms explore the design space globally, the requirements engineering software should provide the capability to grasp and validate the set of requirements as a globally integrated whole. To implement this capability, it is reasonable to employ system multi-modeling tools that allow constructing a formalized conceptual representation of stakeholders, their functions, communication, automation, equipment, and all kinds of relationships between these on the common presentational field. An example of such a tool is ArchiMate [8]. ArchiMate language and framework are intended to compose formal yet graphical descriptions of the enterprise architecture, divided into business, application, and technology layers. In each layer, three aspects are considered: active elements that exhibit behavior (e.g. Process or Function), an internal structure, and elements that define how information is used or communicated. Elements are connected by typed relationships such as "used by", "realization", and "assignment". The ArchiMate syntax allows presenting any elements and relations on the same graph albeit not as profoundly as "polydiagrammatic" system design languages like SysML. Consider the following example of employing ArchiMate in model based engineering of smart power systems [9]. Requirements to the automated electricity consumption accounting system were specified on ArchiMate in the form of models of organizationaltechnical situations involving prosumers interacting with distribution grid dispatchers. From these models, constituents of the system software were automatically generated, including UML diagrams, SQL database specifications, WSDL service descriptions, BPEL process models, HTML user interface forms, and ultimately the C\# source code.

Another approach to requirements consolidation and integration is known as co-modeling data and processes [10]. Upon applying the approach, the model of data that collectively describe the desired power system "shape" is constructed incrementally according to the needs of the energy management processes that operate with them. Requirements engineers elicit structure of processes and associated data model elements simultaneously, either automatically (by the process execution context provided that it has been specified formally) or manually. The structure is composed iteratively in the backward direction from outputs (results) of processes to inputs (source data) until external operations are reached that lay beyond the target system scope. As a result, the data model comes supplied with an exhaustive comprehensive contextual markup by functions of automated processes. The holistic set of structural and behavioral requirements to the power system emerges, with consistency and relevance of the data guaranteed "by construction". To facilitate visual presentation and computer analysis of such holistic sets, a special bimodal (textual and graphical) language OPM was proposed [11]. It has been recently adopted as ISO/PAS 19450:2015 "Automation systems and integration-Object-Process Methodology". It allows representing data model elements as stateful objects linked to processes that create or consume them or change their states.

\section{CONCLUSION}

A generative design technology capable to provide efficiency analysis and generation of design decisions in automatic mode without human intervention is highly relevant for application in critical power supply infrastructure engineering. As shown above, there are a number of techniques, standards, and tools capable to form a considerable basis for development of the technology. The elaboration of the basis in order to create and widely adopt a full-fledged software tool for generative design of power systems is an important direction for future research. 


\section{REFERENCES}

[1] J. Kowalski, "CAD is a lie: generative design to the rescue," San Rafael, CA: Autodesk, 2016. https://www.autodesk.com/redshift/ generative-design/. Accessed July 14, 2019.

[2] Y. Kim, D. Shin, M. Petricca, S. Park, M. Poncino, and N. Chang, "Computer-aided design of electrical energy systems," in Proc. IEEE/ACM ICCAD, ACM, pp. 194-201, 2013.

[3] S. P. Kovalyov, "Domain engineering of distributed measurement systems," Optoelectronics, Instrumentation and Data Processing, vol. 44(2), pp. 125-130, 2008.

[4] S. P. Kovalyov, "Category theory as a mathematical pragmatics of model-based systems engineering," Informatics and Applications, vol. 12(1), pp. 95-104, 2018.

[5] N. B. Ilyichev, E. N. Ilyicheva, and A. I. Kuleshov, "Objectbased modeling for an electrical system design using EnergyCS software," CADmaster, vol. 60, pp. 80-82, 2011. [In Russian].

[6] Z. Shi, R. Wang, and T. Zhang, "Multi-objective optimal design of hybrid renewable energy systems using preferenceinspired coevolutionary approach," Solar Energy, vol. 118, pp. 96-106, 2015.

[7] S. Oh, Y. Jung, I. Lee, and N. Kang, "Design automation by integrating generative adversarial networks and topology optimization," in ASME 2018 International Design Engineering Technical Conferences and Computers and Information in Engineering Conference, American Society of Mechanical Engineers, p. V02AT03A008, 2018.

[8] M. Lankhorst et al, Enterprise Architecture at WorkModelling, Communication and Analysis. 4th edition. Springer, 2017. $360 \mathrm{p}$.

[9] A. V. Amelichkin and S. P. Kovalyov, "Model-based design technology for automated energy management tools," in Proc. XVII PCMCS, Samara, Russia, pp. 167-173, 2015. [In Russian].

[10] S. P. Kovalyov, "Co-modeling energy management data and processes," in Proc. MLSD'2016, Moscow, Russia, vol. II, pp. 28-30, 2016.

[11] D. Dori, Model-Based Systems Engineering with OPM and SysML. Springer, 2016. $411 \mathrm{p}$. 